\title{
Stochastic Stability and Bifurcation of A High Speed Rotor-Bearing System with Random Excitation
}

\author{
Jiarong Lư ${ }^{1}$, Jiangang Zhang ${ }^{1}$, Wenju $\mathrm{Du}^{2}$ and Hongwei Luo ${ }^{1}$ \\ ${ }^{1}$ Department of Mathematics, Lanzhou Jiaotong University, 730070, Anning, Lanzhou, China \\ ${ }^{2}$ School of Traffic and Transportation, Lanzhou Jiaotong University, 730070, Anning, Lanzhou, China
}

\begin{abstract}
In this paper, a high speed rotor-bearing system with stochastic excitation is considered, and study the stability and Hopf bifurcation of the system by using quasi-nonintegrable Hamiltonian system theory. Then, the conditions of local and global stability of system are obtained by largest Lyapunov exponent and boundary category. Finally, the solution of FPK equation can be got, which is stationary probability density function and jointly stationary probability density function, and then, by simulating its graph to illustrate the results.
\end{abstract}

\section{Introduction}

The rotor bearing system involves many nonlinear problems in rotating machinery, and its dynamic behaviors are very complex. Hu and Xian [1] established a mathematical model of an asymmetric double-disc rotor-bearing system and use nonlinear dynamics theory to consider nonlinear oil-film force and rub-impact force. And then, Ninoslav Truhar et al. [2] considered a mechanical system excited by external force with the development of nonlinear dynamics theory, nonlinear dynamics connects with rotor dynamics tightly, which makes the research on rotor dynamics present a new outlook. In the past decade, many researches about the nonlinear stochastic dynamics and controls which have been shifted from Lagrange system to Hamilton system, and the nonlinear stochastic dynamical system is expressed as dissipative Hamilton system with stochastic excitation. Finally, according to the intergrability and resonance of Hamilton system, a theory based on Hamilton system with stochastic excitation is presented and developed, which is used to study mechanical system with stochastic excitation. $\mathrm{Xu}$ et al. [3] discussed the stochastic stability and bifurcation of multiwalled carbon nanotubes-absorbing hydrogen atoms subjected to thermal perturbation. They studied the dynamic behavior by useing the quasi-nonintegrable Hamiltonian system theory and the stochastic parametric excitation is regarded as Gauss white noise. $\mathrm{Li}$ [4] also studies stochastic stability of quasi-integrable and resonant (nonresonant) Hamiltonian systems under parametric excitations of combined Gaussian and Poisson white noises. In this study, the high speed rotor-bearing system with stochastic parametric excitation is presented in section 2. Then, the largest Lyapunov exponent [5] of Ito stochastic differential equation can be obtained by using stochastic averaging method in section 3 . In the section 4 , the stochastic Hopf bifurcation of the system study is studied. Finally, some examples and numerical simulations are given in section 5 .

\section{System model}

A high speed rotor-bearing system [6] is considered. Due to the external random factors and the internal random vibration, then regarding all stochastic excitations as Guass white noise. The equation of motion of the non rotating damped system when the global assembling of the matrices is done is given by

$$
M \ddot{q}(t)+C \dot{q}(t)+K q=f q \xi(t)
$$

where $q(t)$ is a vector containing the generalized coordinates, referred to an inertial frame, $M$ is the symmetric mass, $C$ is the symmetric damping coefficient, $K$ is the symmetric stiffness coefficient, and $f$ is a function of $p$ and $q, \xi(t)$ is the Gauss white noise whose mean is zero and intensity is $2 \mathrm{D}$.

Let $p=\dot{q}, c=C / M, k=K / M$, then the Eq. (1) becomes

$$
\left\{\begin{array}{l}
\dot{q}=p \\
\dot{p}=-c p-k q+f q \xi(t)
\end{array}\right.
$$

The Hamiltonian function of Eq. (2) can be shown as follows:

$$
H=\frac{1}{2} p^{2}+\frac{k}{2} q^{2}
$$


According to the quasi-nonintegrable Hamiltonian system theory, the Hamiltonian function $H(t)$ converges weakly in probability to a one-dimensional Ito diffusion process [7]. The averaged Ito equation about the Hamiltonian function can be shown as follows:

$$
d H=m(H) d t+\sigma(H) d B(t)
$$

where $B(t)$ is standard Wiener process, $m(H)$ and $\sigma(H)$ are drift and diffusion coefficients of Ito stochastic process can be obtained in stochastic averaging method $[8]$.

$$
\begin{aligned}
& m(H)=-c H+\frac{2 D f^{2}}{3 c} H^{2} \\
& \sigma^{2}(H)=\frac{4 D f^{2}}{3 c} H^{2}
\end{aligned}
$$

\section{Stochastic stability}

The local stability of the stochastic system will be discussed in the following, and the largest Lyapunov exponent of Ito stochastic differential equation can be defined as

$$
\lambda=\lim _{t \rightarrow \infty} \frac{1}{t} \ln \left\|Z\left(t, \mathrm{z}_{0}\right)\right\|
$$

then the largest Lyapunov exponent is

$$
\begin{aligned}
\lambda & =\lim _{t \rightarrow \infty} \frac{1}{t} \ln H^{1 / 2}(q, p)=\left[\dot{m}(0)+(\dot{\sigma}(0))^{2} / 2\right] / 2 \\
& =-\frac{c}{2}
\end{aligned}
$$

By Oselesec multiplicative ergodic theorem [9], the condition that the trivial solution of system keeps asymptotically stable with probability 1 can be got:

Lemma 3.1. The conditions to keep the system local stable can be obtained: the largest Lyapunov exponent is less than zero, which means $c>0$.

It is remarkable that that largest Lyapunov exponent can judge local stability of the trivial solution but is helpless for global stability. Therefore, using the boundary category of Eq. (4) to study global stability. Then the two boundaries will be mainly discussed in the following, which is the left boundary $H \rightarrow 0$ and right boundary $H \rightarrow \infty$.

Because of $\sigma^{2}(H)=0$ and $m(H)=0$ when $H \rightarrow 0$, then the left boundary $H \rightarrow 0$ belong to the first singular boundary and the drift and diffusion coefficients converge asymptotically to the two equations

$$
m(H)=-c H+\mathrm{O}(H), \sigma^{2}(H)=\frac{4 D f^{2}}{3 c} H^{2}+\mathrm{O}\left(H^{2}\right)
$$

then, the diffusion exponent $\alpha_{l}$, drift exponent $\beta_{l}$ and character value $c_{l}$ of left boundary can be obtained

$$
\begin{aligned}
\alpha_{l} & =2, \beta_{l}=1 \\
c_{l} & =\lim _{H \rightarrow 0^{+}} \frac{2 m(H) H^{\alpha_{l}-\beta_{l}}}{\sigma^{2}(H)} \\
& =\frac{3 c^{2}}{2 D f^{2}}
\end{aligned}
$$

all the solution curves in state space of system is converging asymptotically to left boundary $H \rightarrow 0$ when left boundary $H \rightarrow 0$ is attractive to nature, which means $c_{l}<1$.

Similarly, it can be get that $\sigma^{2}(H)=\infty$ and $m(H)=\infty$ when $H \rightarrow \infty$, then the right boundary $H \rightarrow \infty$ belong to the second singular boundary and the drift and diffusion coefficients converge asymptotically to the two equations

$$
m(H) \rightarrow \mathrm{O}\left(H^{2}\right), \sigma^{2}(H) \rightarrow \mathrm{O}\left(H^{2}\right)
$$

then, the diffusion exponent $\alpha_{r}$, drift exponent $\beta_{r}$ and character value $c_{r}$ of right boundary can be obtained $\alpha_{r}=2, \beta_{r}=2$

$$
c_{r}=\lim _{H \rightarrow \infty} \frac{2 m(H)|H|^{\alpha_{r}-\beta_{r}}}{\sigma^{2}(H)}
$$

Since $\beta_{r}>\alpha_{r}-1$ and $m(+\infty)<0$, we can get the right boundary $H \rightarrow \infty$ is entering boundary.

Lemma 3.2. The conditions to keep system global stable can be obtained: the left boundary $H \rightarrow 0$ is attractive to nature and the right boundary $H \rightarrow \infty$ is entering boundary, which means $3 c^{2} / 2 D f^{2}<1$

\section{Stochastic hopf bifurcation}

There are two kinds of stochastic bifurcations, one is dynamic bifurcation (D-bifurcation), which is used to study the stationary probability density of system response and determined by the value of lyapunov exponent. Another is phenomenology bifurcation (Pbifurcation), which is used to study the joint probability density, and determined by the number, place and shape of peak.

The averaging FPK equation [10] of Eq. (4) is

$$
\frac{\partial f}{\partial t}=-\frac{\partial}{\partial H}[m(H) f]+\frac{1}{2} \frac{\partial^{2}\left[\sigma^{2}(H) f\right]}{\partial H^{2}}
$$

then the solution of Eq. (13) is

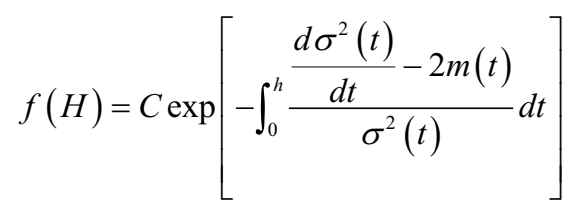


then the stationary probability density function can be got where $\eta=\frac{3 c^{2}}{2 D f^{2}}-2, C$ is normalized constant.

Then, the jointly stationary probability density function also can be got

$$
f(q, p)=C\left[\frac{1}{2} p^{2}+\frac{k}{2} q^{2}\right]^{\eta} \exp \left[-\left(\frac{1}{2} p^{2}+\frac{k}{2} q^{2}\right)\right]
$$

The $f(H)$ is a Delta function when $\eta<-1$, which is a monotone decreasing function and gets maximum value when $-1<\eta<0$, and the first bifurcation will be appeared. The $f(H)$ will still get the maximum value when $\eta>0$ but the maximum value deviates from the origin, and the second bifurcation will be appeared. Then we have the following conclusion from above analysis. The system (12) will undergoes stochastic D-bifurcation when $\eta=-1$, and will undergoes stochastic P-bifurcation when $\eta=0$, finally, the Hopf bifurcation of the system is constituted by these two bifurcations.

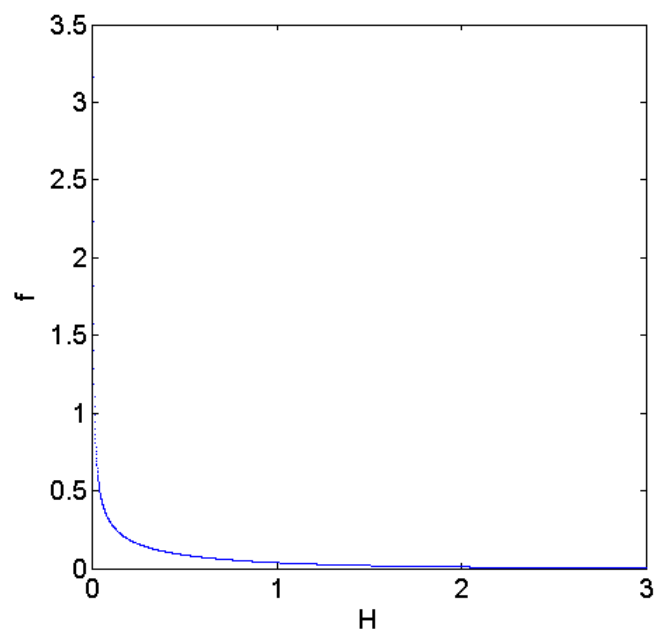

$$
f(H)=C H^{\eta} \exp [-H]
$$

\section{Numerical example}

We choice $c$ as the bifurcation parameter, the qualitative variation of the system response with the change of the parameter $c$ was observed, and mainly discuss the Hopf bifurcation at $\eta=0$, and observe the numerical results of stationary probability density function and jointly stationary probability density function with variation of parameter $c$. We consider the system with the following coefficients: $k=1, D=0.5, f=0.5$.

The images of stationary probability density function and jointly stationary probability density function show in Fig 1-4, when $c$ varies near $c=0.7348$. The stationary probability density changed from Monotone decreasing function to single peak function and Hopf bifurcation appeared. Similarly, the jointly stationary probability density changed from single peak shape to crater-shape and Hopf bifurcation appeared when $c=0.7348$.

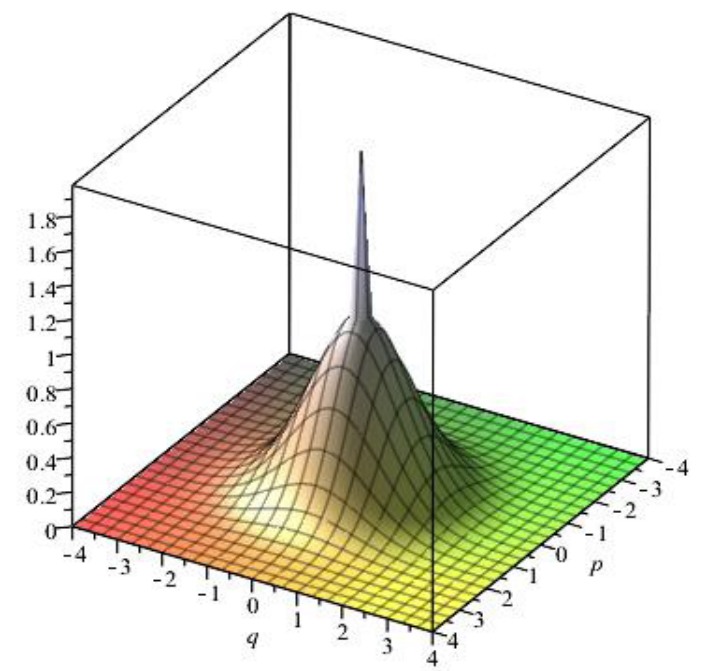

Figure 1. Stationary probability density and jointly stationary probability density when $c=0.5, \eta=-0.67$.
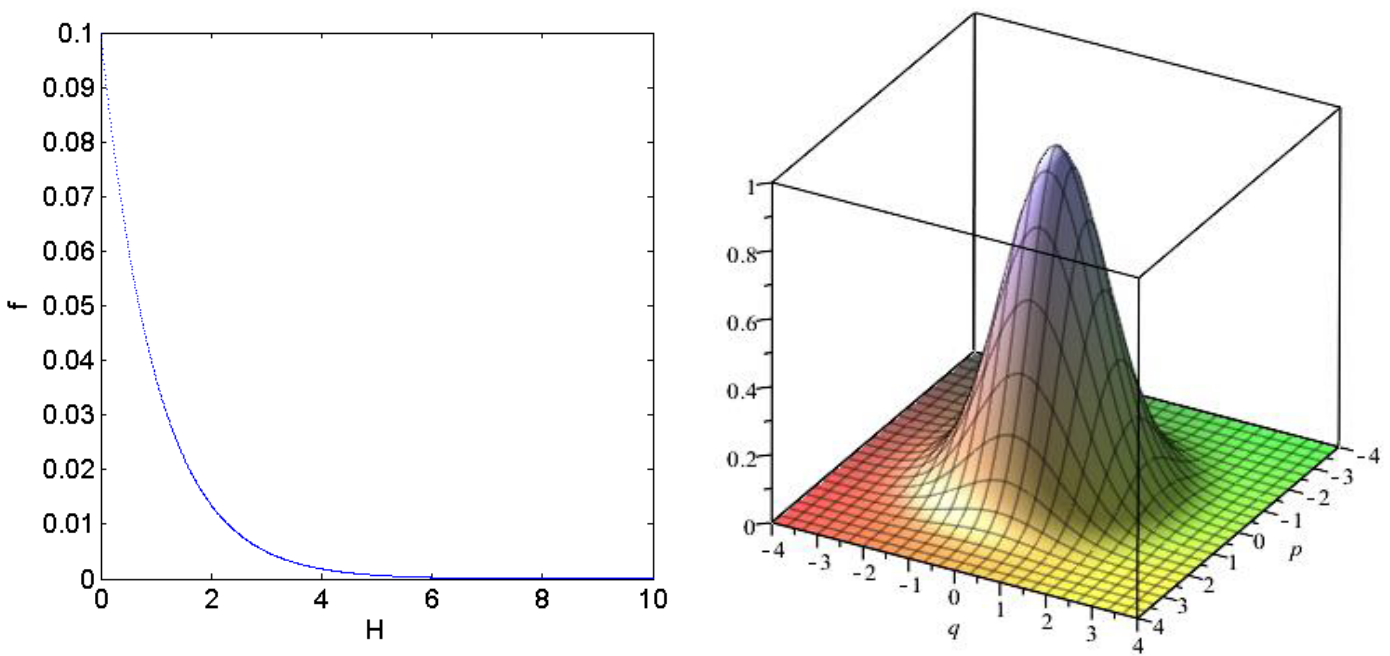

Figure 2. Stationary probability density and jointly stationary probability density when $c=0.7348, \eta=0$. 

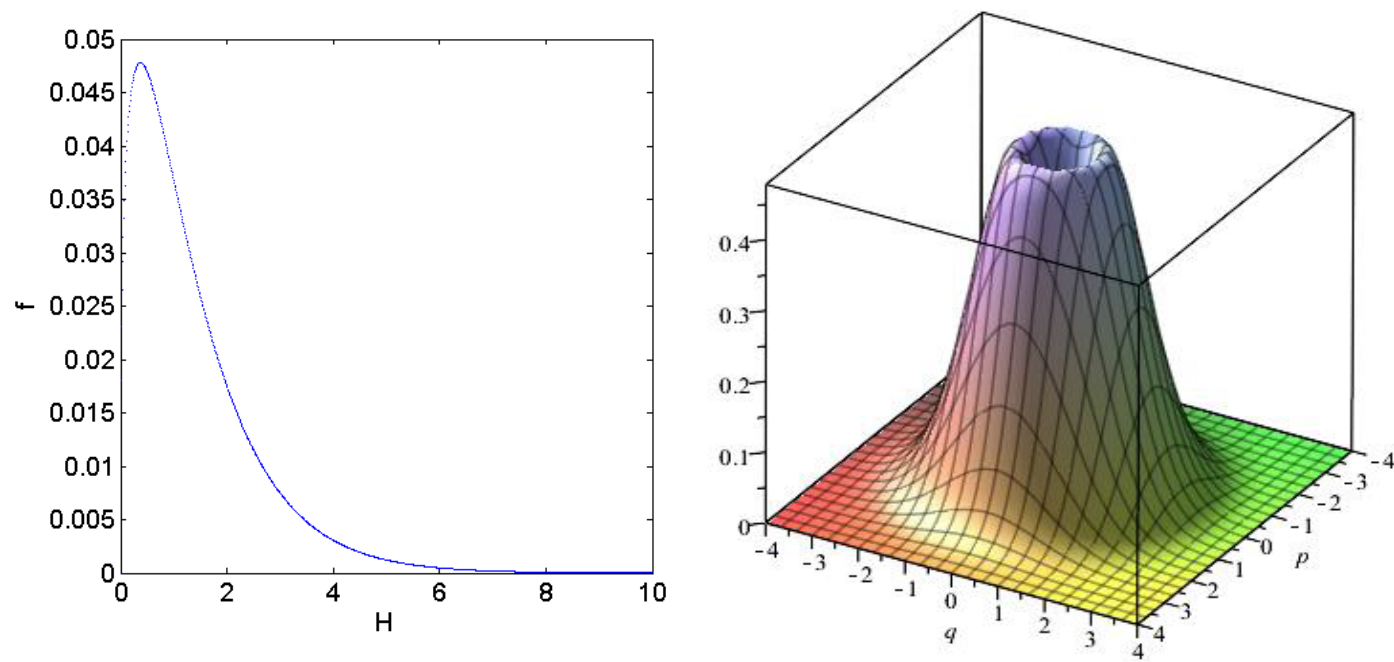

Figure 3. Stationary probability density and jointly stationary probability density when $c=0.8, \eta=0.37$.
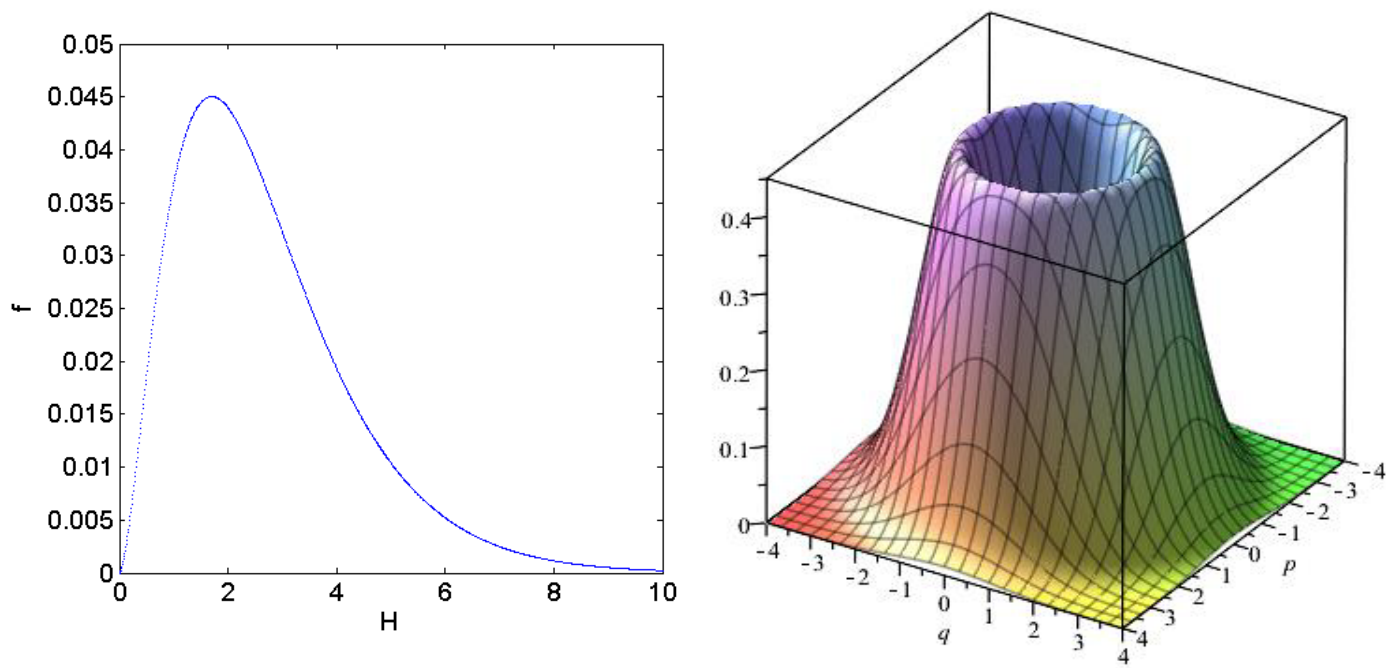

Figure 4. Stationary probability density and jointly stationary probability density when $c=1, \eta=1.7$.

\section{Conclusion}

In this paper, the stochastic stability and Hopf bifurcation of nonlinear damping in a high speed rotor-bearing system with random excitation are discussed by using the method of quasi-nonintegrable Hamiltonian system theory. The local stability of the system can be judged by largest Lyapunov exponent, then, we get the parameter conditions when Hopf bifurcation appears. Finally, some numerical results are given to illustrate the results. In the following study, the stochastic averaging method will be used to transform the Hamilton function into a onedimensional Ito diffusion process, and will establish the reliability function and first-passage time to study the effect of first-passage phenomenon on system characteristics.

\section{Acknowledgment}

This The authors gratefully acknowledge the support from the National Natural Science Foundation (No. 61364001) and Science and Technology Program of Gansu Province (No. 144GKCA018), China.

\section{References}

1. L. Xiang, A. J. Hu, L. L. Hou, Nonlinear coupled dynamics of an asymmetric double-disc rotorbearing system under rub-impact and oil-film forces, Applied Mathematical Modelling 40 (2016) 45054523.

2. N. Truhar, Z. Tomljanovic, K. Veselic, Damping optimization in mechanical systems with external force, Applied Mathematics and Computation 250 (2015) 270-279.

3. J.Z. Xu, D.X. He, X.L. Zhao, Status and prospects of Chinese wind energy, Energy2010;35:4439e44.

4. W.Y. Liu, W.Q. Zhu, Stochastic stability of quasiintegrable and resonant Hamiltonian systems under parametric excitations of combined Gaussian and Poisson white noises, International Journal of NonLinear Mechanics 67 (2014) 52-62.

5. X.D. Gu, W.Q. Zhu, A stochastic averaging method for analyzing vibro-impact systems under Gaussian white noise excitations. JS. Vibration. Vol. 333, No. 9, 2632-2642 (2014). 
6. M.H. Jalali, M. Ghayour, S. Ziaei-Rad, B. Shahriari, Dynamic analysis of a high speed rotor-bearing system, Measurement 53 (2014) 1-9.

7. W.Q. Zhu, Z.L. Huang, Y.Q. Yang, Stochastic averaging of quasi integrable Hamiltonian systems. J. Appl. Mech. Vol. 64, 157-64 (1997).

8. W.T. Jia, W.Q. Zhu, Y. Xu, Stochastic averaging of quasi-non-integrable Hamiltonian systems under combined Gaussian and Poisson white noise excitations, International Journal of Non-Linear Mechanics 51 (2013) 45-53.

9. Pavel Chigansky, An ergodic theorem for filtering with applications to stability, Volume 55, Issue 11, November 2006, Pages 908-917.

10. W.Q. Zhu, Z.L. Huang, Stochastic Hopf bifurcation of quasi nonintegrable Hamiltonian systems, Int J Nonlin Mech 1999; 34: 437-47. 